\title{
MS07-P21 | Structure-Function Characterisation Of Novel Galactosidases From Lactobacillus Plantarum: Exploring The Link Between Gut Bacteria And Lipid
}

\section{LEVELS}

Feliciotti, Irene (University of Reading, Reading, GBR); Kolida, Sofia (Optibiotix HPLC, London, GBR); Rastall, Bob (University of Reading, Reading, GBR); Watson, Kim (University of Reading, Reading, GBR)

The human gut microbiome is a complex ecosystem, which plays a crucial role not only in intestinal health but also significantly impacts on factors such as appetite regulation, cardiovascular health, immunomodulation, mood and neuronal developmental disorders. Current research is suggesting that there may be a link between blood lipid profile regulation and the metabolic activity of certain gut microbiome members, suggesting possible applications in the management of cardiovascular disease.

This project focuses on Lactobacillus plantarum, a GRAS (generally regarded as safe) probiotic strain used to regulate the metabolism of bile acids and impact on coronary disease risk biomarkers such as serum lipid profiles and blood pressure. Recent sequencing of Lactobacillus plantarum has revealed potential novel beta-galactosidase sequences responsible for galacto-oligosaccharide (GOS) synthesis. These enzymes will be targeted for detailed structure-function studies to elucidate their functional role in GOS production. The enzymes will be isolated; X-ray crystallography will be used for their structural characterisation and functional testing will be performed to characterise their GOS production. The resulting GOS products will be isolated, characterized structurally and their cholesterol lowering potential and impact on gut microbiome composition and activity will be tested in batch cultures. Their non-prebiotic effects will be tested in different pathways, using relevant mammalian cell lines.

We anticipate that this work will be valuable for understanding detailed mechanisms of action of specific galactosidase enzymes in the synthesis of oligosaccharides with the ability to enhance the numbers and activity of the parent strain and improve human health by targeting specific health biomarkers. 